\title{
ANATOMIA DAS VIAS BILIARES EM DOADORES E RECEPTORES DE TRANSPLANTE HEPÁTICO INTER VIVOS
}

\author{
Renato Vianna SOARES, Júlio Cezar U. COELHO, Jorge E. F. MATIAS, \\ Alexandre C.T. de FREITAS, Clementino ZENI-NETO e José Luis de GODOY
}

RESUMO - Racional - Variações anatômicas das vias biliares são freqüentes e aumentam as complicações dos transplantes hepáticos. Objetivo - Avaliar a anatomia das vias biliares nos doadores e receptores do transplante hepático inter vivos realizados no Hospital de Clínicas da Universidade Federal do Paraná e do Hospital Nossa Senhora das Graças de Curitiba, Curitiba, PR. Casuística e Métodos - A avaliação foi retrospectiva de março de 1998 até setembro de 2002 (23 transplantes), quando os dados passaram a ser captados de forma prospectiva (17 transplantes), até agosto de 2003. Foram obtidos dados de 80 pacientes consecutivos ( 40 doadores e 40 receptores), submetidos a transplante hepático inter vivos, sendo 32 transplantes com receptor adulto e 8 transplantes pediátricos (receptor com idade inferior a 15 anos). Entre os 80 pacientes incluídos no estudo, 51 eram do sexo masculino ( 27 receptores e 24 doadores) e 29 do feminino ( 13 receptores e 16 doadores). A idade média dos doadores foi de 32,6 anos e a dos receptores de 36,3 anos. Resultados - Nas cirurgias de captação do lobo hepático direito, a via biliar direita foi única em 25 entre 29 casos $(86,2 \%)$, dupla em dois $(6,9 \%)$ e tripla em dois $(6,9 \%)$. Nos oito casos em que o setor lateral esquerdo ou o lobo hepático esquerdo foram captados, a via biliar foi única. Entre os 33 pacientes submetidos a colangiografia por ressonância magnética, variações foram verificadas em três pacientes $(9,1 \%)$, sendo a mais comum o ducto biliar posterior direito tributário do ducto hepático esquerdo $(6,06 \% ; n=2)$. Conclusão - A prevalência de anomalias biliares nos transplantes inter vivos de fígado é elevada e algumas não são diagnosticadas com os exames de imagem pré-operatórios.

DESCRITORES - Vias biliares, anatomia \& histologia. Transplante de fígado. Doadores vivos.

\section{INTRODUÇÃo}

O transplante ortotópico de fígado é uma terapia eficiente para a insuficiência hepática terminal. A principa dificuldade encontrada em todo o mundo pelos centros que realizam transplantes hepáticos é o número limitado de doadores, em função do número crescente de pacientes que aguardam transplante. Técnicas inovadoras como a divisão hepática e o transplante hepático com doadores vivos vêm sendo utilizadas para diminuir essa escassez, principalmente na população pediátrica ${ }^{(14,20)}$. O primeiro transplante inter vivos foi realizado por RAIA et al. ${ }^{(18)}$, em 1989, e em 1990, STRONG et al. ${ }^{(24)}$ realizaram o primeiro transplante hepático inter vivos com êxito. HASHIKURA et al. ${ }^{(10)}$, em 1994, realizaram o primeiro transplante inter vivos entre adultos, utilizando o lobo esquerdo do fígado. Inicialmente, essa modalidade de transplante era indicada para crianças e adultos em condições graves e em países onde o número de doadores cadáveres era pequeno ou inexistente ${ }^{(10)}$. Em nosso meio, as técnicas cirúrgicas nos transplantes com doadores vivos foram divulgadas por CARONE et al. ${ }^{(2)}$, em 1997. Complicações de origem biliar são significativas para receptores e até mesmo para doadores nos transplantes hepáticos inter $\operatorname{vivos}^{(5,17)}$. Número expressivo dessas complicações pode ser atribuído a falhas no reconhecimento de anomalias anatômicas $^{(1,7,21)}$.

Variações anatômicas nas vias biliares são freqüentes ${ }^{(11,19)}$. Apenas 55\% da população apresentam a chamada anatomia "normal" (3, 6, 26). O objetivo do presente estudo foi avaliar a anatomia das vias biliares em doadores e receptores de transplantes inter vivos de fígado.

\section{MATERIAIS E MÉTODOS}

A amostra utilizada compreendeu doadores e receptores dos transplantes hepáticos inter vivos feitos no Hospital de Clínicas da Universidade Federal do

Trabalho realizado no Serviço de Transplante Hepático da Universidade Federal do Paraná, Curitiba, PR.

Endereço para correspondência: Dr. Júlio Coelho - Rua Bento Viana, 1140 - apt.2202 - 80240-110 - Curitiba, PR. - E-mail: juliocoelho@bbs2.sul.com.br 
Paraná e no Hospital Nossa Senhora das Graças, Curitiba, PR, durante o período de março de 1998 até agosto de 2003. A avaliação foi retrospectiva de março de 1998 até setembro de 2002 (23 transplantes), quando os dados passaram a ser captados de forma prospectiva (17 transplantes).

$\mathrm{Na}$ avaliação retrospectiva, foram revisados os protocolos de estudo de doadores e receptores de transplante hepático inter vivos. Esses protocolos continham dados dos pacientes, desde anamnese e exame físico, até exames laboratoriais e de imagem. Observações intra-operatórias da anatomia hepática foram compiladas, principalmente em relação à anatomia das vias biliares.

$\mathrm{Na}$ avaliação prospectiva as informações dos doadores e receptores foram computadas à medida que os procedimentos foram sendo realizados. Foram também registrados dados em relação a sexo, idade e sobre a anatomia arterial do fígado obtida em colangiografias pré-operatórias. As anomalias encontradas durante o ato cirúrgico foram compiladas e documentadas por meio de fotografias.

As operações para captação dos órgãos compreenderam ressecções dos segmentos II e III de Couinaud para pacientes pediátricos com peso abaixo de $15 \mathrm{~kg}$; ressecções dos segmentos II, III, IV para pacientes pediátricos com maior peso e ressecções dos segmentos V, VI, VII e VIII para o transplante inter vivos com receptor adulto.

Este estudo foi aprovado pela comissão de ética do Hospital de Clínicas da Universidade Federal do Paraná (protocolo CEP/ HC 759.178/2003-11).

\section{RESULTADOS}

Em 41 transplantes, foram obtidos dados de 82 pacientes. Foram 9 transplantes pediátricos (receptores com idade inferior a 15 anos) e 32 adultos. Foi incluído no estudo um caso de transplante dominó. Nos casos restantes, os doadores e receptores eram relacionados. Foram excluídos do estudo dois

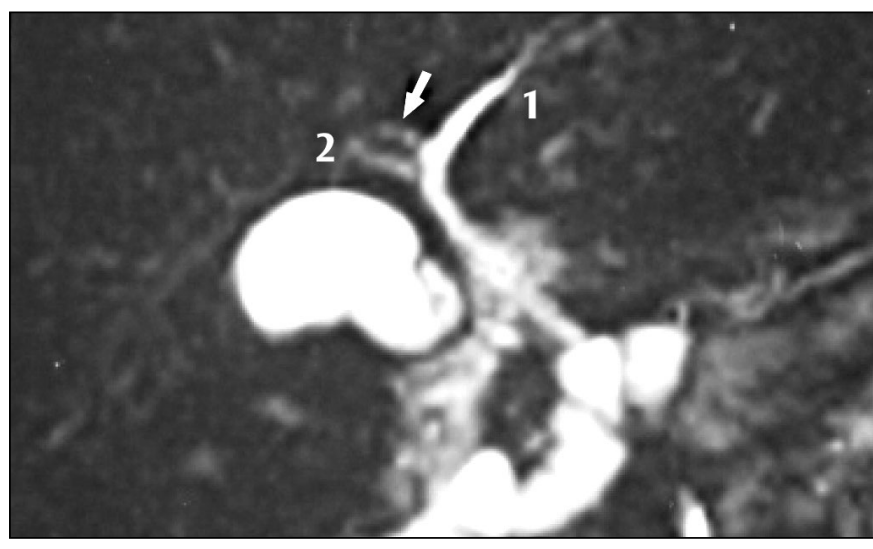

Seta - ducto hepático posterior direito anômalo. 1 - ducto hepático esquerdo. 2 - ducto hepático direito

FIGURA 1 - Colangiorressonância magnética evidenciando um ducto hepático posterior direito tributário do ducto hepático esquerdo. Pode-se também observar que o ducto hepático esquerdo é longo. O ducto hepático direito se une ao ducto hepático esquerdo, logo abaixo desse ducto anômalo pacientes (um receptor pediátrico e um doador para transplante pediátrico), pelo fato do prontuário não ter sido localizado. Em dois casos, o transplante foi suspenso em razão de anomalias venosas significativas no doador, que não foram diagnosticadas no pré-operatório e que poderiam predispor à trombose da veia porta e colocar em risco a vida do doador. Estes dois casos foram incluídos no estudo.

Portanto, de um total de 82 pacientes, foram incluídos no estudo 80 pacientes: 40 doadores e 40 receptores.

A idade dos pacientes variou entre 8 meses e 67 anos. A média de idade foi de 34,5 anos. Cinqüenta e um pacientes eram do sexo masculino $(63,8 \%)$. A média de idade entre os homens foi de 37,2 anos. Pacientes do sexo feminino somaram 29 casos (36,3\%), com média de idade de 29,7 anos.

Em relação aos 40 doadores, 16 eram do sexo feminino (média de idade de 33,6 anos) e 24 eram do sexo masculino (média de idade de 31,5 anos). A média de idade dos doadores foi de 32,6 anos.

Em relação aos 40 receptores, 27 eram do sexo masculino (média de idade de 41,8 anos) e 13 eram do sexo feminino (média de idade de 24,9 anos). A média de idade dos receptores foi de 36,3 anos.

Foram utilizados três tipos de enxertos: segmentos II e III de Couinaud em cinco, segmentos II, III, IV de Couinaud em três e segmentos V,VI,VII e VIII de Couinaud em 29 casos.

\section{Variações anatômicas dos ductos biliares}

Variaçóes anatômicas dos ductos biliares diagnosticadas nos exames de imagem

Entre os 80 pacientes do estudo, 33 doadores foram submetidos a colangiografia por ressonância magnética, para estudo anatômico das vias biliares. Em três pacientes $(9,1 \%)$ foram encontradas anomalias. Em dois casos $(6,06 \%)$, o ducto posterior direito foi tributário do ducto hepático esquerdo (Figura 1); em outro caso $(3,03 \%)$, houve junção dos ductos hepáticos direito, anterior e posterior, com o ducto esquerdo (via biliar trifurcada no hilo hepático). Entre seis receptores que foram submetidos a ressonância magnética do abdome na investigação da suas doenças hepáticas, foi diagnosticado cisto de colédoco tipo IV em um caso.

Variaçóes anatômicas dos ductos biliares diagnosticadas nas operaçóes

Entre os 80 pacientes do estudo, 8 receptores tinham doenças primárias das vias biliares como causa de suas falências hepáticas, não possibilitando uma análise anatômica. Foram, então, analisadas as configurações das vias biliares de 72 pacientes, sendo 40 doadores e 32 receptores. Foram descritas 8 anomalias $(11,1 \%)$ : 6 ocorreram entre os 40 doadores $(15 \%)$ e 2 entre os 32 receptores $(6,25 \%)$.

As anomalias encontradas entre os doadores foram: em um paciente $(2,5 \%)$, o ducto biliar posterior direito unia-se ao ducto biliar esquerdo; em um paciente $(2,5 \%)$, o ducto biliar direito era trifurcado; em um paciente $(2,5 \%)$, o ducto posterior direito era ramo do ducto hepático comum (Figura 2); em um paciente $(2,5 \%)$, o ducto posterior e anterior direito se uniam ao ducto esquerdo. Em dois pacientes (5\%), foi visualizado um ducto biliar 
fino proveniente do setor posterior direito. As duas anomalias anatômicas verificadas em receptores foram a bifurcação do ducto hepático comum no hilo hepático $(6,52 \%)$.

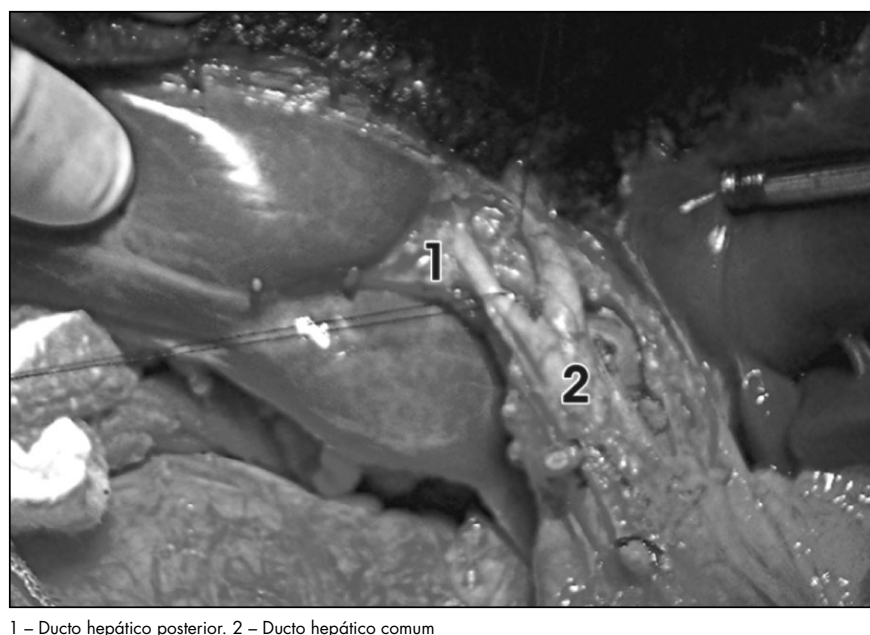

FIGURA 2 - Fotografia obtida no ato operatório de um doador evidenciando o ducto hepático posterior direito tributário do ducto hepático comum. Após a captação do lobo direito havia três ductos biliares para serem reconstruídos

Nos cinco casos nos quais foi captado o setor lateral esquerdo (segmentos II e III) e nos três casos em que o lobo esquerdo foi captado (segmentos II, III, IV), a via biliar era única. Nos 29 casos de captação do lobo direito (segmentos V, VI, VII, VII), a via biliar foi única em 25 pacientes, dupla em dois e tripla em dois (Tabela 1). Em dois doadores de lobo direto, foram identificados ramos biliares de pequeno calibre, não passíveis de reconstrução.

TABELA 1 - Número de ductos biliares reconstruídos

\begin{tabular}{lcccc}
\hline Lobo & Segmentos captados & $\begin{array}{c}\text { N. }{ }^{\circ} \text { de ductos biliares } \\
\text { reconstruídos }\end{array}$ & $\begin{array}{c}\text { N. }{ }^{\circ} \text { de } \\
\text { pacientes }\end{array}$ & $\%$ \\
\hline \multirow{2}{*}{ Esquerdo } & II,III & 1 & 5 & 100,00 \\
& II,III,IV & 1 & 3 & 100,00 \\
& V,VI,VII,VIII & 1 & 25 & 86,2 \\
Direito & V,VI,VII,VIII & 2 & 2 & 6,9 \\
& V,VI,VII,VIII & 3 & 2 & 6,9 \\
\hline $\mathrm{n}=37$ & & & &
\end{tabular}

Comparação da anatomia das vias biliares observada nos exames de imagem e nas operaçóes

Analisando os 33 pacientes (doadores) submetidos a colangiografia por ressonância magnética, em 6 houve variações na anatomia das vias biliares $(18,18 \%)$, na cirurgia ou na colangiografia (Tabela 2). Em três pacientes com colangiografias normais, foram encontradas variações durante as operações $(9,1 \%)$. Em um receptor em que a ressonância nuclear magnética sugeria cisto de colédoco tipo IV, verificou-se na operação que a via biliar era normal.

\section{DISCUSSÃO}

A incidência de complicações biliares é maior após transplante hepático inter vivos ou "split-liver", quando comparados com transplante de fígado total ${ }^{(4,9)}$. Entre $10 \%$ a $30 \%$ dos transplantes cadavéricos têm complicações biliares e a anastomose biliar foi considerada o tendão de Aquiles do transplante hepático ${ }^{(13)}$. Em séries que avaliam as complicações biliares nos transplantes inter vivos, os índices são variáveis: FAN et al ${ }^{(8)}$ reportaram $43 \%$ de complicações nos primeiros 37 pacientes operados em seu grupo e $8 \%$ nos 37 pacientes subseqüentes; ICOZ et al. ${ }^{(13)}$ obtiveram $30 \%$ de complicações em 50 casos estudados; HISATSUNE et al. ${ }^{(12)}$ reportaram $35 \%$ de complicações em 73 casos.

Diferenças anatômicas existem entre as vias biliares dos dois lobos hepáticos. $\mathrm{O}$ ducto hepático esquerdo é mais longo que o direito e menos sujeito a malformações, e em $90 \%$ dos casos de doação de lobos hepáticos esquerdos e segmentos laterais esquerdos, um orifício único para reconstrução pode ser obtido $^{(15)}$. No presente estudo, uma via biliar única foi obtida nos oito casos de captação de segmento lateral esquerdo ou do lobo hepático esquerdo.

A árvore biliar direita, por sua vez, sofre ramificação precoce e é sujeita a um número maior de variações. Considerando o estudo do COUINAUD ${ }^{(6)}$, o ducto biliar direito é único em $57 \%$ dos indivíduos e, segundo YOSHIDA et al. ${ }^{(27)}$, a via biliar direita é única em $67,7 \%$. Em nosso meio, em estudo realizado por CHAIB et al. ${ }^{(4)}$ em cadáveres, os ductos biliares do lobo direito foram múltiplos em 78,3\% dos casos. Nesta série, entre os 32 casos de doação do lobo direito, a via biliar direita foi única em 28 pacientes $(87,25 \%)$, dupla em dois $(6,25 \%)$ e tripla em dois $(6,25 \%)$. Em dois pacientes $(6,25 \%)$ foram identificados ductos biliares de fino calibre, não passíveis de implante. Nos relatos de transplantes hepáticos de lobo

TABELA 2 - Comparação da anatomia das vias biliares observada nos exames de imagem e nas operações

\begin{tabular}{llll}
\hline N. ${ }^{\circ}$ paciente & Doador & $\begin{array}{l}\text { Colangiografia por } \\
\text { ressonância magnética }\end{array}$ & Cirurgia \\
\hline 34 & doador LD & Normal & Ducto posterior direito tributário ducto hepático comum \\
59 & doador LD & Normal & Ducto biliar fino proveniente do setor posterior direito(*) \\
45 & doador LD & Junção dos ductos anterior e posterior direitos com o ducto esquerdo & Presença de três ductos no lobo hepático direito \\
23 & doador LD & Normal & Junção dos ductos anterior e posterior direitos \\
& & & com o ducto esquerdo \\
71 & doador LD & Ducto posterior direito tributário ducto esquerdo & Ducto posterior direito tributário ducto esquerdo \\
75 & doador LD & Ducto posterior direito tributário ducto esquerdo & Ducto biliar fino proveniente do setor posterior direito \\
\hline
\end{tabular}

(*) Nestes dois casos, os ductos biliares foram ligados 
direito, a anatomia da via biliar direita apresenta resultados conflitantes. TESTA et al. ${ }^{(25)}$ publicaram estudo com 30 doadores e concluíram que orifícios únicos podem ser obtidos após a divisão hepática em aproximadamente $50 \%$ dos indivíduos. FAN et al. ${ }^{(8)}$ obtiveram via biliar única em 63 , dentre 74 casos de captação de lobo direito $(81,08 \%)$, dupla em $10(12,34 \%)$ e tripla em 1 caso $(1,23 \%)$. ICOZ et al. ${ }^{(13)}$ obtiveram via biliar única em 29 (58\%), dentre 50 lobos direitos captados, em 17 a via biliar direita era dupla (34\%) e em 4 pacientes, tripla $(8 \%)$. SETTMACHER et al. ${ }^{(22)}$ obtiveram via biliar única em 27 , dentre 50 casos (54\%) de captação do lobo direito, dupla em 20 casos $(40 \%)$ e tripla em $3(6 \%)$. Se a distância entre os orifícios for pequena, estes podem ser reunidos por meio de ductoplastia, possibilitando a realização de somente uma anastomose ${ }^{(16,22)}$.O número de anastomoses biliares é fator de risco para complicações e por isso estudo anatômico deve ser realizado para que a divisão da via biliar seja no ponto mais adequado possível, buscando a obtenção de um único orifício para reconstrução.

A possibilidade de se excluir um potencial doador antes da operação em razão de anatomia biliar aberrante é a razão principal da realização de exames de imagem pré-operatórios. A colangiografia por ressonância magnética sendo utilizada com freqüência crescente, por ser exame não-invasivo e de elevada exatidão. Na avaliação de potenciais doadores em transplantes hepáticos inter vivos, a colangiorressonância magnética apresentou sensibilidade de $66,67 \%$ e especificidade de $100 \%$ na detecção de anomalias biliares neste estudo, em comparação com os achados das operações. KIM et al. ${ }^{(16)}$ realizaram estudo similar e a colangiografia por ressonância magnética teve sensibilidade e especificidade de $92,3 \%$ e $100 \%$, respectivamente, em comparação com a colangiografia intraoperatória. Estima-se que 3\% da população seja impedida de doar o lobo direito em razão de anomalias biliares ${ }^{(6)}$.

A isquemia é causa importante de fístulas e estenoses biliares no transplante hepático. Nos transplantes inter vivos, tem relevância a vascularização dos ductos hepáticos direito e esquerdo. Esses ductos são vascularizados por um plexo derivado diretamente das artérias hepáticas direita e esquerda e ramos acessórios (quando presentes), e indiretamente da artéria gastroduodenal via plexo que vasculariza a via biliar principal ${ }^{(16,23)}$. Desta forma, é importante a preservação do plexo periductal no procedimento de doação com secção dos ductos biliares próximos à superfície hepática. $\mathrm{Na}$ cirurgia do receptor, deve se ter atenção para não haver lesão dos plexos arteriais, derivados da artéria gastroduodenal e da artéria hepática direita $^{(15)}$.

Conclui-se que a prevalência das anomalias nas vias biliares é elevada nos transplantes inter vivos de fígado e que algumas anomalias não são diagnosticadas nos exames de imagem pré-operatórios.

Soares RV, Coelho JCU, Matias JEF, Freitas ACT, Zeni-Neto C, Godoy JL. Study of biliary anatomy in living related liver transplantation. Arq Gastroenterol. 2006;43(3):173-7.

ABSTRACT - Background - Anatomic variations of the biliary tree are frequent and increase complications after liver transplantation. Aim - To describe the anatomy of the bile ducts of donors and recipients of living related liver transplantation. Methods - From March 1998 to September 2002, the study was retrospective (23 transplantations). From October 2002 to August 2003, the study was prospective (17 transplantations). We studied the hepatic anatomy of 80 consecutive patients ( 40 donors and 40 recipients) of the living-related liver transplantation program of the "Hospital de Clínicas da Universidade Federal do Paraná" and the "Hospital Nossa Senhora das Graças", Curitiba, PR, Brazil; 51 were male ( 27 recipients 24 donors) and 29 female (13 recipients and 16 donors). The median age among the donors was 32.6 years and among the recipients was 36.3 years. Thirty-two recipients were adults and 8 recipients were under 15 years old. The bile duct anatomy was studied by magnetic resonance cholangiography in 33 patients, and anomalies were seen in 3 of them (9.1\%). Results - The most prevalent variation of bile ducts was the fusion of the right posterior duct with the left duct $(6.06 \% ; n=2)$. In the 40 harvesting operations, the right bile duct was single in 25 patients $(87.5 \%)$, among the 32 right-lobe donors, double in $2(6.25 \%)$ and triple in $2(6.25 \%)$. All of the eight left livers procured had single bile ducts. Among the 40 recipients, the common bile duct was bifurcated in 2 of them (5\%). Conclusion - The prevalence of biliary anomalies is high in patients subjected to living liver transplantation and some anomalies are not diagnosed with preoperative imaging exams.

HEADINGS - Bile ducts, anatomy \& histology. Liver transplantation. Living donors. 


\section{REFERÊNCIAS BIBLIOGRÁFICAS}

1. Ayuso JR, Ayuso C, Bombuy E, De Juan C, Llovet JM, De Caralt TM, Sanchez M, Pages M, Bruix J, Garcia-Valdecasas JC. Preoperative evaluation of biliary anatomy in adult live liver donors with volumetric mangafodipir trisodium enhanced magnetic resonance cholangiography. Liver Transpl. 2004;10:1391-7.

2. Carone E, Chapchap P, Pugliese V, Averbach M, Abdalla R, Saad R. Transplante hepático com doador vivo familiar: técnica operatória no doador. Rev Col Bras Cir. 1997;24:235-40.

3. Chaib E, Bertevello P, Pinto FCG, Ferreira CEH, Nagahama CI, Cahali IB, Perini MV, Xavier ACG, Costa LJM. Principais variações do sistema biliar extra-hepático e sua aplicação na técnica de transplante de fígado chamada split-liver. Rev Hosp Clín Fac Med Univ São Paulo. 1995;50:311-3.

4. Chaib E, Ribeiro MAF, Saad WA, Gama-Rodrigues J. The main hepatic anatomic variations for the purpose of split-liver transplantation. Transpl Proc. 2005;37:1063-6.

5. Coelho JCU, Matias JEF, Baretta GAP, Celli A, Pisani JC, Yokochi JM. Complicações biliares pós-transplante hepático intervivos. Rev Col Bras Cir. 2005;32:195-200.

6. Couinaud C. Le foie, études anatomiques et chirurgicales. Paris: Masson; 1957.

7. Desphande RR, Heaton ND, Rela M. Surgical anatomy of segmental liver transplantation. Br J Surg. 2002;89:1078-88.

8. Fan ST, Lo CM, Liu CL, Tso WK, Wong J. Biliary reconstruction and complication of right lobe live-donor liver transplantation. Ann Surg. 2002;236:676-83.

9. Freitas ACT, Coelho JCU, Parolin M, Matias JF, Zeni Neto C, Gonçalves CG. Fatores de risco e conduta nas complicações do trato biliar no transplante hepático. Rev Col Bras Cir. 2000;27:359-65.

10. Hashikura Y, Makuuchi M, Kawasaki S, Matsunami H, Ikegami T, Nakazawa Y, Kiosawa $\mathrm{K}$, Ichida T. Successful living-related partial liver transplantation to an adult patient. Lancet. 1994;343:1233-4.

11. Healey JE, Schroy PC. Anatomy of biliary ducts within the human liver; analysis of prevailing pattern of branching and major variations of biliary ducts. Arch Surg. 1953;66:599-616.

12. Hisatsune H, Yazumi S, Egawa H, Asada M, Hasugawa K, Kodama Y, Okazaki K, Itoh K, Takakuwa H, Tanaka K, Chiba T. Endoscopic management of biliary stricture after duct-to-duct biliary reconstruction in rigth-lobe living donor liver transplantation. Transplantation. 2003;76:810-5

13. Icoz G, Kilik M, Zeytunlu M, Celebri A, Ersoz G, Killi R, Nemis A, Karasu Z, Yuzer Y, Tokat Y. Biliary reconstructions and complications encountered in 50 consecutive right - lobe living donor liver transplantation. Liver Transpl. 2003;9:575-80.

14. Kasahara M, Egawa H, Tanaka K, Ogawa K, Uryuhara K, Fujimoto Y, Ogura Y, Ued $\mathrm{M}$, Takada Y, Tanaka K. Variations in biliary anatomy associated with trifurcated portal vein in right-lobe living-donor liver transplantation. Transplantation. 2005;79:626-7.
15. Kawashi S, Shimazu M, Wakabayashi G, Hoshino K, Tanabe M, Yoshida M, Morikawa Y, Kitajima M. Biliary complications in adult living donor liver transplantation with duct-to-duct hepaticocholedochostomy or Roux-en-y hepaticojejunostomy biliary reconstruction. Surgery. 2002;132:48-56.

16. Kim RD, Sakamoto S, Haider M, Molinari D, Gallinger S, Mcgilvray SD, Greig PD, Grant DR, Cattral MS. Role of magnetic resonance cholangiography in assessing biliary anatomy in right lobe living donors. Transplantation. 2005;79:1417-21.

17. Lee VS, Morgan GR, Lin JC, Nazzaro CA, Chang JS, Teperman LW, Krinsky GA. Liver transplant donor candidates: associations between vascular and biliary anatomic variants. Liver Transpl. 2004;10:1049-54.

18. Raia S, Nery JR, Mies S. Liver transplantation from live donors. Lancet. 1989;8661:497.

19. Reichert PR. Contribuição ao estudo das complicações biliares nos transplantes de fígado de tamanho reduzido [tese]. São Paulo: Faculdade de Medicina da Universidade de São Paulo; 1998

20. Renz JF, Reichert PR, Emond JC. Biliary anatomy as applied to pediatric living donor and split-liver transplantation. Liver Transpl. 2000;6:801-4.

21. Renz JF, Roberts JP. Long term complications of living donor liver transplantation. Liver Transpl. 2000;6:s73-6.

22. Settmacher U, Steinmuller TH, Schmidt SC, Heise M, Pascher A, Theruvath T, Hintze R, Neuhaus P. Technique of bile duct reconstruction and management of biliary complications in right lobe living donor liver transplantation. Clin Transpl. 2003;17:37-42.

23. Stapleton GN, Hickman R, Terblanche J. Blood supply of the right and left hepatic ducts. Br J Surg. 1998:85:202-7.

24. Strong RW, Lynch SV, Ong TH, Matsunami H, Koido Y, Balderson GA. Successful liver transplantation from a liver donor to her son. N Engl J Med. 1990;322:1505-7.

25. Testa J, Malagó M, Gamazo CV, Lindell G, Broelsh CE. Biliary anastomosis in living related liver transplantation using the right liver lobe: techniques and complications. Liver Transpl. 2000;6:710-4.

26. Varotti G, Gondolesi GE, Goldman J, Wayne M, Florman SS, Schwartz ME, Miller $\mathrm{CM}$, Sukru E. Anatomic variations in right liver living donors. J Am Coll Surg. 2004;198:577-82.

27. Yoshida J, Chijiwa K, Yamaguchi K, Yokohata K, Tanaka M. Practical classification of the branching types of the biliary tree: an analysis of 1094 consecutive direct cholangiograms. J Am Coll Surg. 1996;182:37-40. 